\title{
Evolution of Clusters in Cold plus Hot Dark Matter Models
}

\author{
Charles Walter and Anatoly Klypin \\ Department of Astronomy \\ New Mexico State University \\ Las Cruces, NM 88003-0001
}

\begin{abstract}
We use N-body simulations to study evolution of galaxy clusters over the redshift interval $0 \leq \mathrm{z} \leq 0.5$ in cosmological models with a mixture of cold and hot dark matter $(\mathrm{CHDM})$. Four different techniques are utilized: the cluster-cluster correlation function, axial ratios and quadrupoles of the dark matter distribution in individual clusters, and virial properties. We find that the correlation function for clusters of the same mass limit was larger and steeper at high redshifts. The slope increases from 1.8 at $z=0$ to 2.1 at $z=0.5$. Comoving correlation length $r_{c}$ scales with the mass limit $M$ within comoving radius $1.5 h^{-1} \mathrm{Mpc}$ and the redshift $z$ as $r_{c} \approx 20(1+z)\left(M / M_{*}\right)^{1 / 3}$, where $M_{*}=3 \times 10^{14} h^{-1} M_{\odot}$. When the correlation length is normalized to the mean cluster separation $d_{c}$, it remains almost constant: $r_{c} \approx(0.45-0.5) d_{c}$. For small masses of clusters $\left(M<2 \times 10^{14} h^{-1} M_{\odot}\right)$ there is an indication that $r_{c}$ goes slightly above the relation with the constant of proportionality being $\approx 0.55-0.6$.

Anisotropy of density distribution in a cluster shows no change over redshift with axial ratios remaining constant around 1.2. In other words, clusters at present are as elongated as they were at the epoch of their first appearance. While the anisotropy of clusters does not change with time, the density profile shows visible evolution: the slope of density profile changes from $\gamma \approx-3.5$ at $z=0.5$ to $\gamma \approx-2.5$ at the present. We find that the core of a cluster remains essentially the same over time, but the density of the outlying regions increases noticeably. The virial relation $M \sim v^{2}$ is a good approximation, but there is a large fraction of clusters with peculiar velocities greater than given by this relation, and clusters with the same rms velocities have smaller masses in the past, a factor of 2 at $z=0.5$.
\end{abstract}

Subject headings: cosmology: theory - dark matter-large-scale structure of the universe - galaxies: clustering-methods: numerical 


\section{Introduction}

The distribution of galaxy clusters can provide a great deal of insight as to the structure of the universe. By comparing with real data such as the Abell (Abell 1958), APM (Dalton et al. 1992), or Edinburgh-Durham (Nichol et al. 1992) cluster catalogues, the validity of many cosmological models can be determined. While in the past we were mainly interested in properties of clusters at $\mathrm{z}=0$, new observational results will bring more information on the evolution of cluster properties. Among interesting tests there are "traditional" tests like evolution of the correlation function and the mass function. It is also interesting to look for more detailed information. Statistics like elongation of clusters and density profiles could shed light on the rate of evolution of fluctuations and thus the mean density of the universe and the nature of dark matter.

Many different cosmological models have been proposed to address these issues. Among these are the standard CDM model, CDM with a cosmological constant $\Omega_{\Lambda}=0.5-0.8$, and the CHDM model with 20-30\% of mass in the form of the hot dark matter (presumably massive neutrinos). The CHDM model (for references see Klypin et al. (1993)) was shown to fit reasonably well available data on cluster properties at $z=0$. For example, Holtzman \& Primack (1993) using the peaks formalism for gaussian density fields found that the correlation function of clusters in the CHDM model is consistent with the correlation function of Abell clusters. Later their results were confirmed by N-body simulations for Abell clusters (Jing et al. 1993, Klypin \& Rhee 1994, hereafter KR94; but see also Cen \& Ostriker 1994) and for APM clusters (KR94, Dalton et al. 1994). Borgani et al. (1994, 1995) came to the same conclusion using the Zeldovich approximation. The CHDM model predicts (e.g., KR94, Borgani et al. 1994) the cluster mass function in agreement with the results of White et al. (1993) and Biviano et al. (1993) for Abell clusters. The problem of abundance of clusters with different masses can be addressed using other approaches. Bartlett \& Silk (1993), KR94, and Jing \& Fang (1994) estimated the distribution function of gas temperature in galaxy clusters using either the Press-Schechter approximation or N-body results in conjunction with temperature-velocity dispersion relation. The temperature distribution function was estimated by Bryan et al. (1994) based on N-body plus hydrodynamic simulations. The velocity dispersion function is another way to measure the abundance of different clusters (Jing \& Fang 1994). All the tests favor the CHDM model. The real problem for the model is related with the amount of gas in clusters. Estimates based on X-ray observations indicate that the ratio of baryonic mass to the total cluster mass is in the range $M_{\mathrm{b}} / M_{\text {tot }}=0.1-0.25$ for the assumed Hubble constant $H_{0}=50 \mathrm{~km} / \mathrm{s} / \mathrm{Mpc}$ (White et al. 1993, White \& Fabian 1995). This 
is significantly larger than the upper limit $\Omega_{b}<0.1$ predicted from primordial nucleosynthesis calculations (Walker et al. 1991, Krauss \& Kernan et al. 1994). CHDM models with two or three neutrinos can slightly ease the problem because those neutrinos are too hot and avoid the central $\sim 1 \mathrm{Mpc}$ region of galaxy clusters. This results in decreasing of the amount of dark matter in the central parts of clusters by at most 20-30\%, increasing the expected ratio of baryons to dark matter to $0.12-0.13$.

The standard CHDM model with $30 \%$ of mass in neutrino has severe problems in explaining the amount of neutral gas in high redshift $(z>3)$ damped Ly- $\alpha$ clouds (Klypin et al. 1995 and references therein). More promising versions of the CHDM scenario with less mass in the hot component (Ma \& Bertschinger 1994, Klypin et al. 1994, Primack et al. 1995) predict significantly more high redshift objects, making the model compatible with observational data. At the same time, the new variants of CHDM keep the shape of spectrum and the amplitude of fluctuations on clusters scale 10-100 Mpc almost the same as in the old CHDM model, which still makes interesting the analysis of clusters in the old CHDM model.

The correlation function of galaxy clusters is still an interesting and controversial test. Recent estimates of the correlation length for Abell clusters give $r_{c} \approx 20-22 h^{-1} \mathrm{Mpc}$ (e.g., Peacock \& West 1992; Postman, Huchra, \& Geller 1992), which is slightly less than the old estimate $r_{c} \approx 25 h^{-1} \mathrm{Mpc}$ of Bahcall \& Soneira (1983) and Klypin \& Kopylov (1983). Results of the APM survey show systematically lower values $r_{c} \approx 13-14 h^{-1} \mathrm{Mpc}$ (Dalton et al. 1992, 1994). The difference was attributed to either inhomogeneities in the selection of Abell clusters (e.g. Sutherland 1988) or to the difference in the richness or in the number density of Abell and APM clusters (Bahcall \& West 1992). The dependence of $r_{c}$ on mass and number of clusters is not well defined either in observational data or in theoretical models. Bahcall \& Burgett (1986), Bahcall \& Cen (1992), and Bahcall \& West (1992) argued that $r_{c}=0.4 d_{c}$, where $d_{c}$ is estimated as $\mathrm{n}_{c}^{-1 / 3}, n_{c}$ being the number density of clusters. The situation with observational results is unclear. If we take APM results (Dalton et al. 1992, 1994), EDCC results (Nichol et al. 1992), and estimates for Abell clusters (Bahcall \& West 1992), then all the data seems to be consistent with the relation $r_{c}=0.4 d_{c}$. But without the Abell clusters, the APM and EDCC results do not indicate the scaling and are consistent with no dependence on the mean separation. Unfortunately, the range for $d_{c}$ is quite small in this case, so it is difficult to say if there is a contradiction between those two results. From the theoretical side, the problem is not easier. A dependence of $r_{c}$ on $d_{c}$ or mass of clusters was found for different cosmological models (e.g., Borgani et al. 1994, KR94, Croft \& Efstathiou 1994). But none of the discussed cosmological models show a perfect $r_{c} \propto d_{c}$ relation (Croft \& Efstathiou 1994; Borgani, Coles, \& Moscardini 1994). Moreover, results from different simulations and different 
methods are somewhat contradictory.

In spite of the fact that many problems of physics of present-day clusters are not solved, it is quite interesting to find out what kind of changes one expects for high-z clusters. There are some observational results on those clusters (e.g. Gunn 1990, Luppino \& Gioia 1995, Thimm \& Belloni (1994), Castander et al. 1994). The situation is not clear. There are indications that some of the high-z clusters could be just projection effects (Thimm \& Belloni 1994). Some of them are real, but not very bright (Nichol et al. 1994, Castander et al. 1994). At the same time, it seems that there exist X-ray luminous clusters already at $z \approx 0.5$, which can pose significant problems for cosmological models.

This paper is organized as follows. In section 2 the methods of simulating the clusters and the criteria for cluster selection are described. The evolution of the correlation function over redshift is covered in section 3. Section 4 details two different methods for measuring shapes of clusters and section 5 evaluates the evolution of virial properties.

\section{Simulations and cluster finding algorithm}

Most of the results presented in this paper are based on three N-body simulations. All three were of the standard CHDM model with $\Omega_{C D M}=0.6, \Omega_{\nu}=0.3$, and $\mathrm{h}=0.5$. All simulations

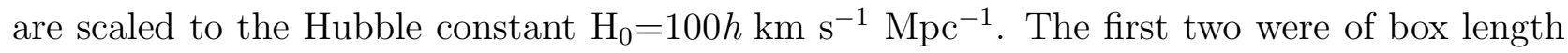
$200 h^{-1}$ Mpc length and are described by Klypin \& Rhee (1994). The third simulation was of a box with length $255 h^{-1} \mathrm{Mpc}$, with a $768^{3}$ mesh, $256^{3}$ cold particles, and $2 \mathrm{x} 256^{3}$ hot particles. This gives a spatial resolution of $0.33 h^{-1} \mathrm{Mpc}$, with each particle having a mass of $5.43 \times 10^{11} h M_{\odot}$. The amplitude of angular fluctuations of the microwave background is chosen in such a way that the quadrupole is normalized to $17 \mu \mathrm{K}$ which is consistent with measurements of COBE for the Harrison-Zel'dovich spectrum. This gives the amplitude of mass fluctuations on a $8 h^{-1} \mathrm{Mpc}$ scale $\sigma_{8}=0.665$. Simulations were run from $z_{0}=13.2$ to the present, with positional, mass, and velocity data saved at three epochs, $z=0,0.16$, and 0.52 . The $200 h^{-1} \mathrm{Mpc}$ boxes have data at $z=0,0.20$, and 0.30 .

In addition, we use the results of one small-box high resolution simulation to address the question of the evolution of cluster internal structure. In this case the simulation was done for a $50 h^{-1} \mathrm{Mpc}$ box with a $800^{3}$ mesh (the resolution is $62.5 h^{-1} \mathrm{kpc}$ ) using $256^{3}$ cold particles and $2 \times 256^{3}$ hot particles. The CHDM model with two equal mass neutrinos accounting for $\Omega_{\text {hot }}=0.20$, baryon density $\Omega_{b}=0.075, h=0.5$, and a CMBR quadrupole of $18 \mu \mathrm{K}$ was used. 
Clusters were determined as maxima of mass within a comoving radius of $1.5 h^{-1} \mathrm{Mpc}$. The choice of the algorithm was motivated by the definition of Abell clusters. We did not try to mimic the observational procedure, specifically, projection effects. This certainly can produce some differences by boosting "observed" mass of clusters. In this paper we neglect effects of projection, which we will address in a separate paper. Figure 1 presents an example of cluster identification. It shows two 2D projections of cold dark matter particles and identified clusters in a $22.5 h^{-1} \mathrm{Mpc}$ cube from the $255 h^{-1} \mathrm{Mpc}$ simulation at $z=0$. The distribution on left panel ( $\mathrm{x}-\mathrm{y}$ projection) indicates that there is a quite large supercluster, which goes almost diagonally from top right corner to bottom left corner. Crowded distribution of a dozen of clusters around $x=220-230 \mathrm{Mpc}$ nicely illustrates the difficulty of identification of cluster in projection when supercluster is seeing edge-on. On the right panel (y-z projection) the supercluster is almost face-on. It is clear that the clump of clusters is really a large filament. The plot also shows that clusters have a strong tendency to be found in most dense regions of the supercluster. For example, a filament at $z=400-420 \mathrm{Mpc}, y=395 \mathrm{Mpc}$ does not have any identified clusters with mass above $2.5 \times 10^{14} h^{-1} M_{\odot}$ while its heavier analog at $y=365 \mathrm{Mpc}$ has six clusters. About half of the clusters found in Figure 1 have visible substructure. The most massive cluster at the top of both panel looks like a double cluster (right panel). Its mass is $2.5 \times 10^{15} h^{-1} M_{\odot}$ and its axial ratio is $a / c=1.4$ (see section 4 ). There is no obvious tendency for large clusters to have more substructure as compared with small ones. For example, the second largest cluster on the plot with mass $1.6 \times 10^{15} h^{-1} M_{\odot}$ does look quite roundish (axial ratio 1.1-1.2). Many small clusters show significant deviation from sphericity.

Number density of clusters in our simulations as a function of mass limit compared to the Press-Schechter approximation and results for Abell clusters estimated by Bahcall \& Cen (1992) are shown in Figure 2. If we assume that Abell richness class zero corresponds to a mass limit of $M>2.5 \times 10^{14} h^{-1} M_{\odot}$, then in the $255 h^{-1} \mathrm{Mpc}$ model we found 297,121 , and 13 "Abell" clusters at the three redshifts 0, 0.16 and 0.52. Bahcall \& Cen's results differ from the rest universally by a factor of 1.6 in mass. There are several possibilities for this inconsistency as discussed by Klypin \& Rhee (1994). For instance, a large portion of the total cluster mass comes from the outer cluster regions. Even in the Coma cluster uncertainties in the total mass do not accurately identify the correct model. The mass ranges from $0.65 \times 10^{15} h^{-1} M_{\odot}$ from Bahcall \& Cen to $1.1 \times 10^{15} h^{-1} M_{\odot}$ from White et al. (1993).

\section{Evolution of correlation function of clusters}


The correlation function can be useful to describe clustering on different scales. We examine the correlation function over time. At the higher redshifts there are very few clusters that meet our fiducial estimate $M>2.5 \times 10^{14} h^{-1} M_{\odot}$ for the mass of a richness zero cluster. So in order to get enough clusters to do meaningful statistics we used half the mass limit just for this analysis. Evolution for the $200 h^{-1} \mathrm{Mpc}$ box is shown in figure 3 . Figure 4 is for the bigger box, and it shows the same trend of increasing correlation function at greater redshifts. This implies that clustering increased in the past, while the number of clusters dramatically decreased. This result can be understood better when the mean separation in comoving coordinates of clusters is considered. Since the number density of clusters decreases with increasing redshift, it is instructive to compare the mean separation $\mathrm{d}_{c}$ with the correlation length $\mathrm{r}_{c}$, defined by computing a power law $\alpha$ over the region $2.5-25 h^{-1} \mathrm{Mpc}$ : $\xi=\left(r / r_{c}\right)^{-\alpha}$. The Levenburg-Marquardt method for finding the minimum in $\chi^{2}$ space is used to solve simultaneously for the correlation length $r_{c}$ and the slope $\alpha$ (Press et al. 1992). In figure 5, correlation lengths are plotted as a function of mass limit. This displays the effects which different mean separations have on clustering. For all redshifts the correlation length increases, showing increasing amplitude of the correlation function for smaller number densities (and larger masses), which can be seen in figures 3-4 at high redshifts. One effect that may be significant if real is the increase in steepness of the slope of the $\mathrm{r}_{c}(\mathrm{M})$ relation with redshift. Figure 6 shows the ratio $\mathrm{r}_{c} / \mathrm{d}_{c}$ as a function of mass limit. This value does not equal 0.4 but remains constant near $0.46 \pm 0.03$ for the $z=0$ case for mean separations ranging from $33.1 h^{-1} \mathrm{Mpc}$ at a mass limit of $2 \times 10^{14} h^{-1} M_{\odot}$ to $49.2 h^{-1} \mathrm{Mpc}$ at a limit of $3.5 \times 10^{14} h^{-1} M_{\odot}$. Comoving correlation length $r_{c}$ scales with the mass limit $M$ within comoving radius $1.5 h^{-1} \mathrm{Mpc}$ and the redshift $z$ approximately as $r_{c} \approx 20(1+z)\left(M / M_{*}\right)^{1 / 3}$, where $M_{*}=3 \times 10^{14} h^{-1} M_{\odot}$.

\section{Shapes of clusters}

We study the evolution of cluster shapes using the $255 h^{-1} \mathrm{Mpc}$ simulation at redshifts $z=0,0.16$, and 0.52. Two separate techniques are used. First, the moment of inertia for each cluster is computed by summing contributions for all cold particles within the radius limit and solving for principal axes. The ratio of the major to minor axis of the inertia tensor is used to characterize the shapes of clusters. Second, we examine the overall quadrupoles $Q_{2}$ of the clusters. We define the multipole $Q_{l}$ as follows (Peebles 1980, section 46): 


$$
Q_{l}^{2}=\sum_{m=-l}^{l} a_{l}^{2}, \quad a_{l}(j)=\frac{\sum_{j=1}^{N} Y_{l}^{m}\left(\theta_{j}, \varphi_{j}\right)}{N}
$$

where $\mathrm{Y}_{l}^{m}$ are spherical harmonics and the summation in the equation is over all dark particles $N$ within the radius limit and over all components $m$ of the multipole. The normalization of the multipoles $Q_{l}$ is defined in such a way that the maximum value of a multipole is 1 , which happens when all particles are colinear. Typical values for a quadrupole $Q=Q_{2}$ are 0.1-0.2. Both statistics are very simple to implement. They test basically the same property, but in a slightly different way.

In Figure 7 we have split each cluster up into two sections, the inner core $\left(r<0.75 h^{-1} \mathrm{Mpc}\right)$ and the outer annulus $\left(0.75 h^{-1} \mathrm{Mpc}<r<1.5 h^{-1} \mathrm{Mpc}\right)$. Poor statistics (only 13 clusters) mar the $z=0.52$ data, but it is clear that in all cases the outer annulus is slightly more anisotropic, suggesting perhaps that relaxation has not been completed. One might expect that new-born clusters at high $z$ would be less relaxed and thus more elongated. Our results indicate that there is no evidence for general trend with the redshift: clusters are as elongated at high $z$ as at present. For both epochs $z=0$ and $z=0.16$ with ample statistics the inner core peaks at an axial ratio of 1.2 and the outer annulus at 1.3. One effect that may show some relaxation over time is the fact that the percentage of clusters with axial ratios $>1.4$ decreases. At $z=0.16$ the inner and outer parts have 17.4 and $37.2 \%$ respectively, whereas at $z=0$ this drops to 11.7 and $22.6 \%$. Figure 8 shows the quadrupole for the same clusters, again with no definite trend. Peaks for the three samples occur at $0.10,0.13$, and 0.17 in increasing redshift. The distribution of quadrupoles is well approximated by the following expression:

$$
\frac{d N}{d Q V}(Q)=\frac{N}{V}\left(\frac{2}{\pi}\right)^{1 / 2} \frac{Q^{2}}{\sigma^{3}} \exp \left(-\frac{Q^{2}}{2 \sigma^{2}}\right),
$$

where $V$ is the total volume; parameter $\sigma$ is related to $\left\langle Q^{2}\right\rangle=3 \sigma^{2}$. The rms value of the quadrupole $\left\langle Q^{2}\right\rangle^{1 / 2}$ is $0.17,0.20$, and 0.20 for the redshift moments $0,0.16$, and 0.52 correspondingly. The difference is not statistically significant.

\section{Evolution of virial properties}

The virial theorem has been shown to be of use in estimating masses of clusters. It predicts for a cluster $M=\alpha R v^{2} / G$, where $v$ is velocity dispersion, $\alpha$ is a numerical coefficient, 
and $R$ is cluster radius. In this paper we take by definition that $R$ is the Abell radius $R_{A}=1.5 h^{-1} \mathrm{Mpc}$. As the result, all details of the cluster structure are hidden in the coefficient $\alpha$. Here are some examples. For a spherical cluster with constant density this parameter is $\alpha=3 / 5$. For the King model $\rho=\rho_{0} /\left[1+\left(r / r_{c}\right)^{2}\right]^{3 / 2}$ with truncated density at some radius $r_{\max }$ the value of parameter $\alpha$ ranges from 0.6 for $r_{\max }<<r_{c}$ to 1.21 at $r_{\max }=10 r_{c}$ to 2.67 at $r_{\max }=50 r_{c}$. The general tendency is clear: larger values of $\alpha$ correspond to more centrally condensed clusters. Figure 9 plots one-dimensional velocity dispersion of dark matter particles against cluster mass for all clusters in the $255 h^{-1} \mathrm{Mpc}$ box with $v>300 \mathrm{~km} \mathrm{~s}^{-1}$. No mass limit is used, so any significant overdensity of cold particles is shown.

The majority of the clusters fall along a line $M \propto v^{2}$ at the top of each graph. For $z=0$ the line gives the value of $\alpha$ equal to 1.1 , which corresponds to $r_{\max }=8 r_{c}$ if we assume the King model, which is in reasonable agreement with our $0.33 h^{-1} \mathrm{Mpc}$ resolution. The existence of the concentration of clusters along the line is quite remarkable. It indicates that the gravitational radius $R_{g} \equiv \alpha R$ is almost the same for clusters with mass $10^{14}-10^{15} h^{-1} M_{\odot}$. All clusters below this line have larger velocity dispersions. This might indicate that (i) their radii are smaller than those of "virialized" clusters on the line, or (ii) their profiles are steeper (resulting in larger values of $\alpha$ ), or (iii) significant degree of merging activity. At first sight the latter option would seem to be the explanation, but inspection of few cases with high velocity and low mass shows that it might not be true. First, the most massive clusters (mass larger than $\left.5 \times 10^{14} h^{-1} M_{\odot}\right)$ do not deviate that wildly from the $M \propto v^{2}$ line. At the same time it does not mean that they do not show significant merging or substructure. For example, the largest cluster in Figure 1 consists of two clusters in the process of merging, but altogether it does not show significant deviation from the $M \propto v^{2}$ line. Second, we found that clusters with large $v$ are often those, which are close to very large nearby clusters or are placed between massive clusters. It seems that tidal field might significantly distort them.

It is interesting to note that while there are many clusters with mass well below that what should be expected for virialized clusters with a given velocity, there are no clusters with mass above the virial relation. This is especially remarkable if we would try to apply the usual top-hat model for cluster formation. It nicely explains why most massive clusters are on the $M \propto v^{2}$ line: their overdensity within an Abell radius is above the 170 that is predicted by the model. But clusters with mass $10^{14} h^{-1} M_{\odot}$ have an overdensity of only 25 . If the model would be correct those are clusters which only recently passed through their turn-around radius and thus should have a velocity smaller than their virial velocity. This would place them above the $M \propto v^{2}$ line. In our simulations we found none of those cases. It seems that the model works reasonably well for massive clusters, which dominate their local environment, but fails 
for small clusters, which are dominated by this environment.

In all three epochs the solid line in Figure 9 is the fit for the $z=0$ case. With increasing redshift the fit for each epoch (shown as a dashed line) moves down, showing that a cluster with the same mass had higher velocity dispersion in the past. This is due to a change in the overall shape of the cluster from core-dominated at $z=0.52$ to having a larger contribution from outer regions in the present epoch. With more of the cluster mass near the core in the past, it would be easier to have an increased velocity. In order the check this, we studied the density profile in most massive clusters. Those with one dimensional velocity dispersions between 855 and $1000 \mathrm{~km} \mathrm{~s}^{-1}$ are considered for their density profiles as shown in Figure 10. The average density profile has been computed at the two extreme redshifts. For the $z=0$ nineteen clusters were used and four clusters for $z=0.52$. Near the center the profiles are unresolved and look similar. But the dropoff in density happens at relatively large radii and it is numerically resolved. It appears to be much steeper for the clusters at large redshifts. Two dashed lines with power laws approaching that of the outer regions are drawn alongside for comparison. The dash-dot-dash line shows a fit to the profiles. We used King approximation for the fits: $\rho /<\rho>\propto\left[1+\left(r / r_{c}\right)^{2}\right]^{-\alpha / 2}$. For $z=0$ this fit gives $\alpha \approx 3$ and for $z=0.52$ the slope is $\alpha \approx 5$.

In order to check that these results are not affected by the finite resolution of our numerical simulations, we traced the evolution of two most massive clusters in our five times higher resolution simulation (box size $50 h^{-1} \mathrm{Mpc}$, resolution $62.5 h^{-1} \mathrm{kpc}$ ). Figure 11 shows the distribution of cold dark matter inside $10 h^{-1} \mathrm{Mpc}$ box around one of the clusters at different redshifts. The cluster is slightly above the center of the box at $z=0$. There is another (smaller) cluster few a megaparsecs away. The fraction of particles shown for $z=0$ is twice smaller than for the other moments. At $z=1$ the cluster is much smaller and its center is at $x=73 \mathrm{Mpc}, y=62 \mathrm{Mpc}$ (note that the axes are not scaled with $h$ ). At $z=2$ the cluster is at the same position, but it is merely a very large halo with mass around $10^{12} M_{\odot}$. It is interesting to note how drastically the distribution of matter changes with time. At $z=0$ most of the halos (tiny knots of particles seeing in the Figure) are found very close to the clusters with some indication of a filament going vertically on the plot. At $z=0.5$ the distribution is dominated by group-size objects. The filament is more prominent and there are some halos away from the center. At $z=1$ the filament is the most prominent structure. Groups are smaller, but still visible. Many halos are outside the filament. At $z=2$ the filament has not formed yet. There are many small size filaments and relatively large halos.

Figure 12 shows the density profile of two large clusters $\left(M=4-5 \times 10^{14} h^{-1} M_{\odot}\right)$ at $z=0$ and $z=1$. Bottom panels present the overdensity of cold dark matter particles with a 
distance scale given in comoving coordinates. Dashed curves are analytical fits of the form $\delta_{0} /\left(1+\left(r / r_{c}\right)^{2}\right)^{\alpha / 2}$. For both clusters we used the same core radii and slopes. At $z=0$ we found $r_{c}=0.070 h^{-1} \mathrm{Mpc}$ and $\alpha=2.30$. For $z=1$ the fits gave $r_{c}=0.085 h^{-1} \mathrm{Mpc}$ and $\alpha=2.70$. The core radii are still not resolved. The slope of the density profile indicates the same trend as in our low resolution simulations (though smaller differences): steeper profiles in the past. In order to directly compare profiles at different redshifts we plot the absolute density in units of mass of hydrogen atom per unit volume in the top panels. Radius is plotted in proper coordinates (the virial theorem requires a proper, not a comoving radius). Now the difference in slopes is more clear. The very central part of the cluster was better resolved in the past: in proper coordinates the resolution at $z=1$ is twice that at $z=0$. This explains why density is higher at the center at $z=1$. In any case, mass within central $100 \mathrm{kpc}$ radius does not change over time. What changes is the density in peripheral parts of the clusters - it increases by an order of magnitude.

While the density in peripheral parts (and, thus, the total mass) evolves quite significantly with time, the velocity dispersion is much more stable. Figure 13 shows three-dimensional velocity profiles for a cluster at $z=0$ and $z=1$ (full curve). Some part of this velocity is due to streaming of material inside the clusters. It is interesting to estimate how large that streaming velocity is when compared with the truly chaotic component. We estimated the chaotic velocity in the following way. We found the velocity of each cold dark matter particle relative to the velocity of cold matter inside a sphere of $150 h^{-1} \mathrm{kpc}$ radius centered on the particle. If there were no streaming velocities, the sphere would not move and the rms relative velocity would be just the same as the rms velocity of all dark matter in the cluster. In the other extreme case, when there are large clumps moving inside clusters with relatively small velocity of internal motion inside the clumps, we would expect that the relative (chaotic) velocity is significantly smaller than the total rms velocity. In reality, the situation is always somewhere in between. The central 0.5 Mpc part seems to be quite relaxed (the two velocities being close), while the peripheral parts show some degree of ongoing evolution. The dashed curves in Figure 13 show the chaotic rms velocity on a $150 h^{-1} \mathrm{kpc}$ scale. At $z=1$ velocities outside the central $0.5 \mathrm{Mpc}$ region are mainly in the streaming component. At $z=0$ the chaotic velocities are much larger and dominate.

\section{Conclusions}


We have studied properties of clusters at different redshifts predicted for the CHDM model. Effects of the correlation function, anisotropies, and density profiles were examined. We find that the correlation function for clusters of the same mass limit was larger and steeper at high redshifts. The slope increases from 1.8 at $z=0$ to 2.1 at $z=0.5$. The comoving correlation length $r_{c}$ scales with the mass limit $M$ within comoving radius $1.5 h^{-1} \mathrm{Mpc}$ and the redshift $z$ as $r_{c} \approx 20(1+z)\left(M / M_{*}\right)^{1 / 3}$, where $M_{*}=3 \times 10^{14} h^{-1} M_{\odot}$. When the correlation length is normalized to the mean cluster separation $d_{c}$, it remains almost constant: $r_{c} \approx 0.45$ $0.5 d_{c}$. For small masses of clusters $\left(M<2 \times 10^{14} h^{-1} M_{\odot}\right)$ there is an indication that $r_{c}$ goes slightly above the relation with the constant of proportionality being $\approx 0.55-0.6$.

Examining isotropies we place rough limits on what is a normal or nearly relaxed cluster. We show that average shapes of clusters do not change significantly over time even in the outer 1-2 Mpc parts of clusters, where the resolution of our simulations is adequate for the problem. There is some evidence showing that the small number of highly anisotropic clusters virialize over time, but more statistics are needed here. Five individual clusters were examined, and those that appear normal to the eye do statistically show to be isotropic, however selection effects on annulus size can make an visually abnormal cluster appear normal statistically. We also found that the cores of clusters (inner $0.5 \mathrm{Mpc}$ ) remain nearly the same for redshifts $z=0-1$ while the outer regions show definite continual accretion and change of slope of the density profile.

Finally, we can see some evidence for evolution by studying virial properties of the clusters. The outlying regions of clusters grow denser at the present epoch and have lower velocity dispersions. Power law fits for density profiles in $\Omega=1$ universes at $z=0$ were shown to range between 2.2 and 2.5, consistent with our results (Crone, Evrard, \& Richstone 1994). Our evidence points to clusters continuing to accrete matter over time, but retaining their basic symmetry.

\section{REFERENCES}

Abell, G. O. 1958, ApJS, 3, 211

Bahcall, N. A., \& Burgett, W.S. 1986, ApJ, 300, L35

Bahcall, N. A., \& Cen, R. 1992, ApJ, 398, L81

Bahcall, N. A., \& Soneira, R. 1983, ApJ, 270, 20

Bahcall, N. A., \& West, M. 1992, ApJ, 392, 419 
Bartlett, G.B., \& Silk, J. 1993, ApJ, 407, L45

Biviano, A., Girardi, M., Giuricin, G., Mardirosian, F., \& Mezzetti, M. 1993, ApJ, 411, L13

Borgani, S., Coles, P., \& Moscardini, L. 1994, MNRAS, 271, 223

Borgani, S., Plionis, M., Coles, P., \& Moscardini, L. 1995, MNRAS, in press

Bryan, G., Klypin, A., Loken, C., Norman, M., \& Burns, J. 1994, ApJ, 437, L5

Castander, F., Ellis, R., Frenk, C., Dressler, A., \& Gunn, J. 1994, ApJ, 424, L79

Cen, R., \& Ostriker, J. 1994, ApJ, 431, 451

Croft, R.A.C., \& Efstathiou, G. 1994, MNRAS, 267, 390

Crone, M.M., Evrard, A.E., \& Richstone, D.O. 1994, ApJ, 434, 402

Dalton, G. B., Efstathiou, G., Maddox, S. J., \& Sutherland, W. 1992, ApJ, 390, L1

Dalton, G. B., Croft, R.A.C., Efstathiou, G., Sutherland, W., Maddox, S. J., \& Davis, M. 1994, MNRAS, 241, L47

Davis, M \& Peebles, P. J. E. 1983, ApJ, 267, 465

Gunn, J., 1990, in Clusters of Galaxies eds. W. Oergerle, et al. , (Cambridge: Cambridge University Press), p.341.

Hauser, M. G., \& Peebles, P. J. E. 1973, ApJ, 185, 757

Holtzman, J. A., \& Primack, J. R. 1993, ApJ, 405, 428

Jing, Y. P., Mo, H. J., Bonner, G., \& Fang, L. Z. 1993, ApJ, 411, 450

Jing, Y. P., \& Fang, L. Z. 1994, ApJ, 432, 438

Klypin, A., Holtzman, J., Primack, J., \& Regos, E. 1993, Astrophys.J., 416, 1

Klypin, A. A., \& Kopylov, A. I. 1983, Soviet Ast., 9, L41

Klypin, A. A., \& Rhee, G. 1994, ApJ, 428, 399

Klypin, A., Borgani, S., Holtzman, J., \& Primack, J. 1995, ApJ, 444, 1

Krauss, L.M., \& Kernan, P.,J. 1994, ApJ, 432, L79

Luppino, G.A., \& Gioia, I.M. 1995, ApJ, in press

Ma, C., \& Bertschinger E. 1994 ApJ, 434, L5

Nichol, R. C., Collins, C. A., Guzzo, L., \& Lumsden, S. L. 1992, MNRAS, 255, 21

Peackock, J.A., \& West, M.J. 1992, MNRAS, 259, 494 
Postman, M., Huchra, J.P., \& Geller, M.J. 1992, ApJ, 384, 404

Press, W. H., Teukolsky, S. A., Vettering, W. T., \& Flannery, B. P. 1992, Numerical Recipes in $\mathrm{C}$, (Cambridge : Cambridge University Press)

Primack, J., Holzman, J., Klypin, A., \& Caldwell, D. 1995, Phys.Rev.Letters 74, 2160

Sutherland, W. J. 1988, MNRAS, 234, 159

Sutherland, W. J. \& Efstathiou, G. 1991, MNRAS, 248, 159

Thimm, G.J., \& Belloni, P. 1994, A\&A, 289, L27

Walker, T.P., Steigman, G., Kang, H., Schramm, D.M., \& Olive, K.A. 1991, ApJ, 376, 51

White, S. D. M., Navarro, J. F., Evrard, A. E., \& Frenk, C. S. 1993, Nature, 366, 429

White, D.A., \& Fabian, A. 1995, MNRAS, 273, 72 
Fig. 1.- Two 2D projections of a box with length $22.5 h^{-1} \mathrm{Mpc}$ from the $255 h^{-1} \mathrm{Mpc}$ sample showing cold dark matter particles. Distances on the plot are scaled to $h=0.5$. Five clusters used as examples are taken from this, ranging in mass from $2.2 \times 10^{14}-1.6 \times 10^{15} M_{\odot}$. Clusters are denoted by circles surrounding the core. The area of the circle is proportional to the mass of the cluster.

Fig. 2.- A comparison of mass functions. The cluster mass function of our $255 h^{-1} \mathrm{Mpc}$ box is shown by the solid curve. Abell clusters estimated by Bahcall and Cen (1992) are displayed two ways. The dotted curve represents their estimation, while the dashed curve represent the mass scale increased by a factor of 1.6. The dot-dashed curve is the mass function predicted by the Press-Schechter approximation with parameter $\delta_{c}=1.5$ and gaussian filter.

Fig. 3.- The two point cluster-cluster correlation function $\xi$ in $\log$ (a) and linear (b) scales for two simulations using $200 h^{-1} \mathrm{Mpc}$ periodic boxes at three different redshifts. Vertical errors are calculated from $\sigma=(1+\xi) / N_{p}^{1 / 2}$, where $\mathrm{N}_{p}$ is the number of pairs counted in a given annulus. Horizontal errors for radii less than $25 h^{-1} \mathrm{Mpc}$ come from $\xi$ and a power law slope computed between 2.5 and $25 h^{-1} \mathrm{Mpc}$. Beyond $25 h^{-1} \mathrm{Mpc}$ horizontal errors show the bin size which is defined as $\Delta \mathrm{r} / \mathrm{r}=0.4$.

Fig. 4.- The same as Figure 2, using a different simulation involving a $255 h^{-1} \mathrm{Mpc}$ box with three times the resolution of the first sample. As in figure 1 there is a large $\xi$ at greater redshift. The $\mathrm{z}=0.52$ curve suffers from poor statistics, but still follows the general trend.

Fig. 5. - Correlation length vs. the mass limit for cluster definition. The correlation length is defined using the power law fit in the region $\left(2.5-25 h^{-1} \mathrm{Mpc}\right)$. The lines for $z=0$ and $z=0.18$ represent averages of data from two $200 h^{-1}$ and one $255 h^{-1} \mathrm{Mpc}$ simulations. The error bars extend from one data point to the other. Typically twice as many clusters in the $255 h^{-1} \mathrm{Mpc}$ simulation meet the mass limit when compared to a $200 h^{-1} \mathrm{Mpc}$ box. The $z=0.3$ comes from the $200 h^{-1} \mathrm{Mpc}$ simulations while the $z=0.52$ comes from the $255 h^{-1} \mathrm{Mpc}$ simulation. 
Fig. 6. - Correlation length normalized to the mean separation between clusters. Since the correlation function and therefore correlation length is a function of number density, an attempt is made at normalization by dividing the correlation length by the mean separation between clusters. While each sample shows some dispersion, an average of similar redshifts shows an expected leveling off. Errors are as in Figure 4.

Fig. 7.- Histogram of the axial ratio between the largest and smallest radii of the cluster. This is determined by transforming the moment of inertia tensor to get the principal axes and solving $I=m r^{2}$ for $r$. The solid line shows the inner volume of the cluster while the dashed line shows the outer annulus. The different epochs are, (a) $z=0.00$, (b) $z=0.16$, (c) $z=0.52$. Each epoch shows the same pattern of greater anisotropy further from the cluster center. The abscissa is normalized by the volume of the box and the binsize. One cluster is equivalent to $1 \times 10^{-6} h^{-1} \mathrm{Mpc}^{-3}$.

Fig. 8.- Distribution of the quadrupole of the cluster anisotropy. Epochs and normalization are as figure 7 . The anisotropy does not appear to vary with redshift. The dashed curves are analytical fits: $(N / V)(2 / \pi)^{1 / 2}\left(Q^{2} / \sigma^{3}\right) \exp \left(-Q^{2} / 2 \sigma^{2}\right)$. Parameter $\sigma$ is related to $\left\langle Q^{2}\right\rangle=3 \sigma^{2}$. The rms value of the quadrupole $\left\langle Q^{2}\right\rangle^{1 / 2}$ is $0.17,0.20$, and 0.20 for the redshift moments 0 , 0.16 , and 0.52 correspondingly. The difference is not statistically significant.

Fig. 9.- Mass vs. velocity at three separate epochs. The solid line is the fit to the $\mathrm{z}=0.0$ case, while dashed lines are fits to the individual epochs. All clusters with velocity dispersions above $300 \mathrm{~km} \mathrm{~s}^{-1}$ are shown.

Fig. 10.- Average density profile for clusters with velocity dispersions between 855 and 1000 $\mathrm{km} \mathrm{s}^{-1}$. The top curve is for $z=0$ and the bottom line for $z=0.52$. Dash-dot-dash curves are fits to the two profiles. Functions are given in the text. Dashed lines are power laws of the given $\gamma$. 
Fig. 11. - Distribution of cold dark matter inside $10 h^{-1} \mathrm{Mpc}$ box around one of the clusters at different redshifts. The cluster is slightly above the center of the box at $z=0$. There is another (smaller) cluster few a megaparsecs away. The fraction of particles shown for $z=0$ is twice smaller than for the other moments.

Fig. 12.- Density profile of two large clusters $\left(M=4-5 \times 10^{14} h^{-1} M_{\odot}\right)$ at $z=0$ and $z=1$. Bottom panels present the overdensity of cold dark matter particles with a distance scale given in comoving coordinates. Dashed curves are analytical fits $\delta_{0} /\left(1+\left(r / r_{c}\right)^{2}\right)^{\alpha / 2}$ with $r_{c}=0.070 h^{-1} \mathrm{Mpc}$ and $\alpha=2.30$ for $z=0$. For $z=1$ the fits gave $r_{c}=0.085 h^{-1} \mathrm{Mpc}$ and $\alpha=2.70$. Top panels show the absolute density in units of mass of hydrogen atom per unit volume. Radius is plotted in proper coordinates.

Fig. 13. - Three-dimensional rms velocity profiles for a cluster at $z=0$ and $z=1$. Full curves are for the total velocity. Some part of this velocity is due to streaming of material inside the cluster. The dashed curves show the chaotic rms velocity on a $150 h^{-1} \mathrm{kpc}$ scale. At $z=1$ velocities outside the central $0.5 \mathrm{Mpc}$ region are mainly in the streaming component. At $z=0$ the chaotic velocities are much larger and dominate. 